\title{
Spin reorientation transition and phase diagram of ultrathin ferromagnetic films
}

\author{
Marianela Carubelli, ${ }^{1 * *}$ Orlando V. Billoni, ${ }^{1, \dagger}$ Santiago A. Pighín, ${ }^{1, \ddagger}$ Sergio A. Cannas, ${ }^{1, \S}$ \\ Daniel A. Stariolo, ${ }^{2, \|}$ and Francisco A. Tamarit ${ }^{1, \pi}$ \\ ${ }^{1}$ Facultad de Matemática, Astronomía y Física, Universidad Nacional de Córdoba, Ciudad Universitaria, 5000 Córdoba, Argentina \\ ${ }^{2}$ Departamento de Física, Universidade Federal do Rio Grande do Sul, Caixa Postale 15051, 91501-979 Porto Alegre, Brazil
}

(Received 14 December 2007; revised manuscript received 25 February 2008; published 7 April 2008)

\begin{abstract}
We show results from Monte Carlo simulations of a two-dimensional Heisenberg model for ultrathin films with perpendicular anisotropy. A complete phase diagram is obtained as a function of anisotropy and temperature, spanning a wide range of behavior. We discuss our results in relation to experimental findings in different ultrathin films. We observe and characterize a line of spin reorientation transitions. This transition from out-of-plane stripe order to in-plane ferromagnetic order presents an intermediate paramagnetic gap in a finite region of parameter space, as reported in experiments. For large anisotropies, direct transitions from a low temperature stripe phase to a paramagnetic or tetragonal phase with dominant perpendicular magnetization is observed, which is also in agreement with experiments. We also show the phase diagram for a system without exchange, i.e., with pure dipolar and anisotropy interactions. It shows a similar behavior to the ferromagnetic case with antiferromagnetic instead of stripe phases at low temperatures. A spin reorientation transition is also found in this case.
\end{abstract}

DOI: 10.1103/PhysRevB.77.134417

PACS number(s): 75.40.Gb, 75.40.Mg, 75.10.Hk

\section{INTRODUCTION}

In recent years, the magnetic behavior of ultrathin films has become of great technological importance due to the applications in magnetic storage devices. As the sizes become smaller and smaller, a detailed microscopic characterization of magnetization processes on the nanometer scale is mandatory. Magnetic order in ultrathin ferromagnetic films is very complex due to the competition between exchange and dipolar interactions on different length scales, together with a strong influence of the shape and the magnetocrystalline anisotropies of the sample. These, in turn, are very susceptible to the growth conditions of the films. ${ }^{1}$ In the past 20 years, a considerable amount of experimental results on different aspects of magnetism in ultrathin films has appeared. Nevertheless, after a careful analysis of the literature, it is difficult to reach general conclusions even in seemingly basic things such as the kind of magnetic order at low temperatures. In view of this complexity, theoretical work on simplified models and computer simulations are essential for rationalizing and guiding new experimental work. In early experiments on $\mathrm{Fe} / \mathrm{Cu}(100)$ films, Pappas et al. ${ }^{2}$ and Allenspach and Bischof ${ }^{3}$ observed a spin reorientation transition (SRT) from a region with perpendicular magnetization to one with in-plane magnetization. In the first experiment, Pappas et al. found a gap with a complete loss of magnetization in between the perpendicular and in-plane phases. Two hypotheses were put forward for the origin of the gap: a dynamic origin, i.e., a loss of long range order due the compensation of perpendicular and in-plane anisotropies in the region around the SRT, and a static one, which is based on a previous theoretical work by Yafet and Gyorgy, ${ }^{4}$ who predicted a perpendicular strip domain configuration as the true ground state of ultrathin films with perpendicular anisotropy. In the second experiment, Allenspach and Bischof discarded the possibility of a completely vanishing magnetization in the vicinity of the SRT but, instead, observed the emergence of stripe magnetic order with a temperature dependent stripe width in general agreement with Yafet predictions. They measured the spontaneous (local) magnetization by using scanning microscopy. In those measurements, no gap was observed between the perpendicular and in-plane phases. On the other hand, a gap over a temperature interval $\Delta T$ $\approx 50 \mathrm{~K}$ appeared when the remanent magnetization, which was approximated by the polarization average over the scan area (and therefore, global), was considered. We will show that, in fact, this kind of behavior, with a direct SRT from a striped to a ferromagnetic in-plane state, is present in a particular anisotropy-temperature region in the phase diagram of our model. Furthermore, the thickness dependence of the SRT temperature observed by Pappas et al. can be qualitatively reproduced by our results on a single monolayer, after noting that the anisotropy behaves as the inverse of the film thickness, as discussed below.

More recently, Won et al..$^{5}$ studied the SRT as a function of temperature and thickness in $\mathrm{Fe} / \mathrm{Ni} / \mathrm{Cu}(001)$ films. They found an exponential decrease of stripe width on approaching the SRT and the possibility of a paramagnetic gap between the out-of-plane stripe phase and the in-plane ferromagnetic phase. The existence of the gap was interpreted by the authors in terms of a crossover between typical dipolar and anisotropy lengths. They defined a Curie temperature as a function of the dipolar length and, depending on it being higher or lower than the SRT temperature, a paramagnetic gap may or may not manifest in the system. Indeed, we will show that also this kind of behavior with a SRT and a gap is present in a particular anisotropy-temperature region in the phase diagram of our model. Although we were not able to quantitatively test the phenomenological arguments of Won et al. because of our too small working stripe width, their conclusions are completely consistent with the scenario that emerges from our simulations. In yet another set of important experiments, Vaterlaus et al. ${ }^{6}$ found evidence of a two step disordering process of perpendicularly magnetized ultrathin $\mathrm{Fe} / \mathrm{Cu}(100)$ films. The films show stripe phases at low tem- 
peratures which lose orientational order and eventually evolve into a "tetragonal liquid phase" with short range stripe order showing $90^{\circ}$ rotational symmetry. This phase further evolves in a continuous way toward a final paramagnetic phase. In these experiments, the films present a strong perpendicular anisotropy and no SRT is observed; magnetization is always out of plane. We will show that this is also observed in our simulations in the parameter region corresponding to strong anisotropy. In this region of the phase diagram, a direct transition from a stripe phase to a paramagnetic (or tetragonal) phase is observed. In the region of strong anisotropy, the thermodynamic phases can be studied in the Ising limit. Detailed ground state calculations ${ }^{7}$ and numerical simulations ${ }^{8-10}$ have been done in recent years, and a successful picture of this region of the phase diagram has emerged. In an extended region of temperatures and anisotropies, MacIsaac et al. ${ }^{11}$ presented a phase diagram of a Heisenberg model with exchange interaction, dipolar interaction, and perpendicular surface anisotropy. Their phase diagram (Fig. 3 of Ref. 11) is similar to our present results. Nevertheless, both diagrams differ in an important result: while they obtained a SRT from a low temperature in-plane ferromagnet to a perpendicular stripe phase at higher temperatures (at variance with most experiments), our results show the inverse trend, i.e., from a perpendicular stripe phase at low temperature to an in-plane ferromagnet or paramagnetic phase at high temperatures, which is consistent with experimental results. Our SRT line is supported by experimental as well as several theoretical arguments as will be explained below.

The nature of the different phase transitions is a delicate issue and several controversial results are spread in the literature. In the present work, we did not pursue to set in a definitive answer, but, nevertheless, we added results to the previous ones. In the high anisotropy limit, our results regarding the nature of the stripe-tetragonal phase are again consistent with similar simulations in Ising systems which point to predominantly first order transitions. This result is again at variance with the continuous transition reported by MacIsaac et al. ${ }^{11}$ on the same region. At intermediate anisotropies, the same transition line gradually changes its behavior and the transition seems to become continuous or weakly first order in the region where a gap is observed around the SRT. This behavior is similar to the phenomenology recently observed in field theoretical models for thin films with Langevin dynamics. ${ }^{12,13}$ Another relevant aspect concerns the possible existence of an intermediate nematic phase as theoretically predicted by Abanov et al. ${ }^{14}$ and recently on more general grounds by Barci and Stariolo ${ }^{13}$ and characterized in Langevin simulations by Nicolao and Stariolo ${ }^{12}$ and also in Monte Carlo simulation of an Ising model by Cannas et al..$^{9}$ In the rest of this work, we will refer to stripe phases regardless of the existence of true long range positional order or only orientational order. Besides the stripe-tetragonal or paramagnetic transition line, we obtained convincing evidence for a first order nature of the SRT line and the continuous nature of the in-plane ferromagneticparamagnetic transition, as will be shown below.

Finally, we also show a complete phase diagram of the pure dipolar system with perpendicular uniaxial anisotropy.

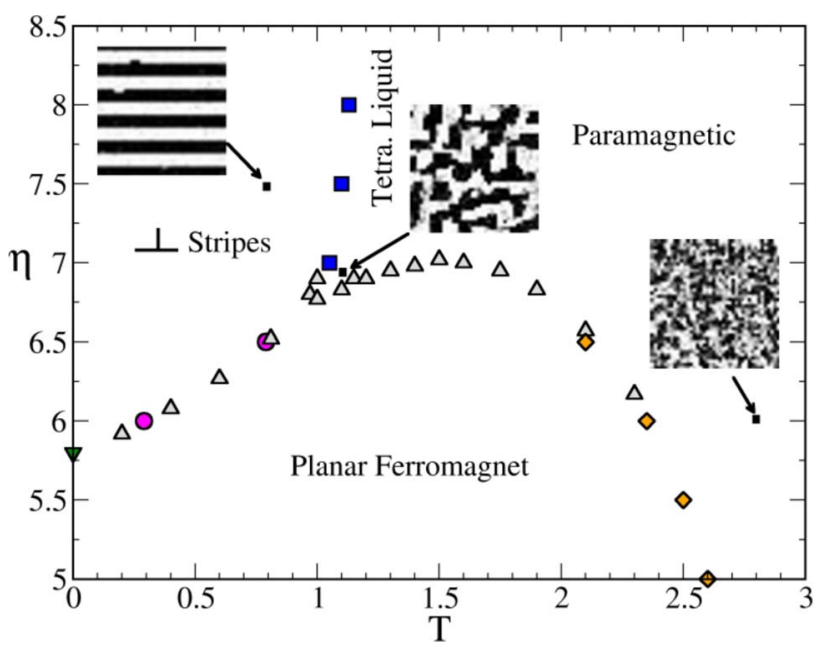

FIG. 1. (Color online) The phase diagram $\eta$ vs temperature for $\delta=3$. The different symbols correspond to different calculation methods; triangle down (green), ground state calculation; circle (red), energy histogram simulations; square (blue), order parameter histogram simulations; triangle up (white), equilibrium and nonequilibrium order parameter simulations (see Sec. II); and diamond (yellow), specific heat simulations. Some typical spin configurations (perpendicular component of the spins) are shown at different phases.

The phase diagram in this limit is similar to the one with exchange interaction, the main difference being the small slope of the SRT line as compared to the ferromagnetic case and the antiferromagnetic nature of the low temperatures phases.

\section{MODEL AND METHODS}

We have performed Monte Carlo (MC) simulations on the usual model Hamiltonian for ultrathin films with exchange, dipolar, and perpendicular anisotropies on a square lattice of side $L=40$ as follows:

$$
\begin{aligned}
\mathcal{H}= & -\delta \sum_{\langle i, j\rangle} \vec{S}_{i} \cdot \vec{S}_{j}+\sum_{(i, j)}\left[\frac{\vec{S}_{i} \cdot \vec{S}_{j}}{r_{i j}^{3}}-3 \frac{\left(\vec{S}_{i} \cdot \vec{r}_{i j}\right)\left(\vec{S}_{j} \cdot \vec{r}_{i j}\right)}{r_{i j}^{5}}\right] \\
& -\eta \sum_{i}\left(S_{i}^{z}\right)^{2},
\end{aligned}
$$

where the exchange and anisotropy constants are normalized relative to the dipolar coupling constant, $\langle i, j\rangle$ stands for a sum over nearest neighbor pairs of sites in the lattice, $(i, j)$ stands for a sum over all distinct pairs, and $r_{i j} \equiv\left|\vec{r}_{i}-\vec{r}_{j}\right|$ is the distance between spins $i$ and $j$. All the simulations were done using the Metropolis algorithm, and periodic boundary conditions were imposed on the lattice by means of the Ewald sums technique. All the results presented in Sec. III refer to the case $\delta=3$, which corresponds, for large values of $\eta$, to a ground state with out-of-plane stripe magnetic structure of width $^{7} h=4$ and to an in-plane ferromagnetic ground state for small anisotropy (see Fig. 1). 
Each spin is defined by a unit vector with components $S^{x}, S^{y}, S^{z}$. The phase diagram has been obtained by measuring the out-of-plane magnetization

$$
M_{z} \equiv \frac{1}{N} \sum_{\vec{r}}\left\langle S^{z}(\vec{r})\right\rangle,
$$

the in-plane magnetization

$$
M_{\|} \equiv \sqrt{\left(M_{x}\right)^{2}+\left(M_{y}\right)^{2}},
$$

and an orientational order parameter similar to the one defined by Booth et al. ${ }^{15}$

$$
O_{h v} \equiv\left\langle\left|\frac{n_{h}-n_{v}}{n_{h}+n_{v}}\right|\right\rangle,
$$

where $\langle\cdots\rangle$ stands for a thermal average, $n_{h}\left(n_{v}\right)$ is the number of horizontal (vertical) pairs of nearest neighbor spins with antialigned perpendicular component, i.e.,

$$
n_{h}=\frac{1}{2} \sum_{\vec{r}}\left\{1-\operatorname{sgn}\left[S^{z}\left(r_{x}, r_{y}\right), S^{z}\left(r_{x}+1, r_{y}\right)\right]\right\}
$$

and a similar definition for $n_{v}$, where $\operatorname{sgn}(x, y)$ is the sign of the product of $x$ and $y$. In the previous definitions, $N=L$ $\times L$ is the number of spins and $M^{x}, M^{y}$ are defined similar to Eq. (2). Other quantities calculated were the specific heat

$$
C \equiv \frac{1}{N T^{2}}\left(\left\langle H^{2}\right\rangle-\langle H\rangle^{2}\right)
$$

and the mean absolute magnetization

$$
P \equiv \frac{1}{N} \sum_{\vec{r}}\left\langle\left|S^{z}(\vec{r})\right|\right\rangle .
$$

In Sec. IV, we calculate the phase diagram for $\delta=0$ (dipolar interactions plus anisotropy). In this case, the relevant phases at low temperatures are antiferromagnetic (AF). For high values of the anisotropy, the ground state is AF with sublattice magnetization and all the spins oriented perpendicular to the plane. ${ }^{16}$ For low values of the anisotropy, the ground state is a highly degenerated planar AF state; the different configurations of this state are described in Ref. 17. To characterize the perpendicular AF state, we calculated the staggered perpendicular magnetization

$$
M_{s \perp} \equiv \frac{1}{N}\left\langle\left|\sum_{\vec{r}}(-1)^{r_{x}+r_{y} S^{z}(\vec{r})}\right|\right\rangle .
$$

To characterize the planar AF state, we calculated the following orientational order parameter ${ }^{16,17}$ :

$$
M_{s \|} \equiv \frac{1}{N}\left\langle\left|\sum_{\vec{r}}(-1)^{r_{y}} S^{x}(\vec{r}) \hat{x}+(-1)^{r} S^{y}(\vec{r}) \hat{y}\right|\right\rangle .
$$

To obtain the phase diagrams $T$ vs $\eta$, we analyzed the behavior of the above quantities by fixing $\eta$ and varying $T$, or vice versa. Those curves were calculated by using two different simulation protocols.

To analyze equilibrium properties, we use a ladder proto$\mathrm{col}$, where the system is initialized at some configuration close to the equilibrium one (either the corresponding ground state at low temperatures or the paramagnetic one at high temperatures) and the independent parameter ( $\eta$ or $T$ ) is varied at discrete steps. The initial configuration for every value of the independent parameter was taken as the last one of the previous value; then we discarded the first $t_{e}$ Monte Carlo steps (MCSs) needed for equilibration and calculated the averages over the next $t_{m}$ MCS. A MCS is defined as a complete cycle of $N$ spin update trials, according to the Metropolis algorithm. Typical values of $t_{e}$ were around $10^{5}$ MCS, while typical values of $t_{m}$ were between $10^{3}$ and $10^{4}$ MCS.

To analyze the possible existence of hysteresis effects, we used a "cooling-heating" procedure, varying the temperature (or $\eta$ ) according to a linear protocol $T(t)=T(0) \pm r t$, where $T(0)$ is the initial temperature, $t$ is measured in MCS, and $r$ is a constant rate. Before starting the protocol, we let the system equilibrate during $t_{e}$ MCS from some appropriate initial configuration (as in the previous protocol) and then we recorded the quantities of interest as a function of time along a complete path to the final temperature; then we repeated the procedure several times, averaging the whole curves over different sets of initial configurations (in the cases were they are random) and over different sequences of thermal noise; typical sample sizes range between 50 and 100 .

\section{PHASE DIAGRAM FOR THE FERROMAGNETIC MONOLAYER}

In Fig. 1, we show the phase diagram in the $(\eta, T)$ plane. The different transition lines were obtained by measuring more than one quantity as is indicated in the figure with different symbols.

As can be seen, all lines are smooth, which gives confidence to the quality of the data. We can clearly distinguish three different phases: perpendicular stripes for low temperatures and strong anisotropy, in-plane ferromagnet for low temperatures and weak anisotropy, and paramagnetic behavior for high temperatures. The disordering of the stripe phase with temperature evolves through a region where the orientational order is lost, but a lower symmetry to $90^{\circ}$ rotations survives and continuously evolves to the complete paramagnetic state. We will call this region tetragonal phase although there is no clear evidence that a sharp transition to a paramagnetic phase with full rotational symmetry is present at high temperatures.

\section{A. Stripe-tetragonal transition}

In Fig. 1, we see that for $\eta>7$, the system goes through a phase transition from a phase with perpendicular stripe order, with stripes of width $h=4$ lattice spacings, to a high temperature tetragonal phase. This transition line has been obtained by calculating histograms of the order parameter $O_{h v}$. One such histogram for $\eta=7.5$ and three characteristic temperatures is shown in Fig. 2. Clear evidence of a first order transition is given by the behavior of the histogram that shows two metastable phases (stripes and tetragonal), which change the stability around the transition temperature $(\approx T=1.1$ in this case). This is at variance with results by MacIsaac et 


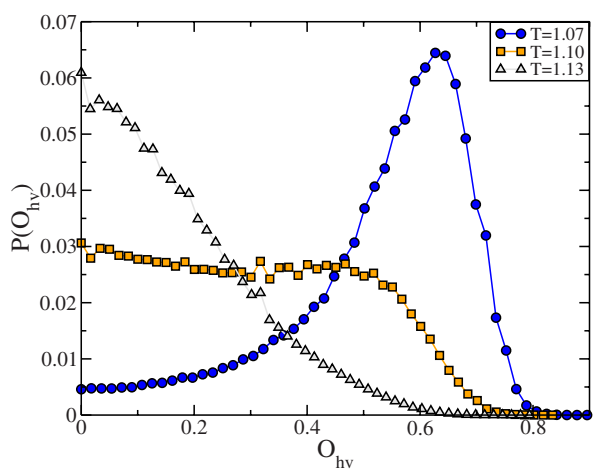

FIG. 2. (Color online) Order parameter $O_{h v}$ per spin histograms for $\eta=7.5$ and different temperatures. The histograms were calculated for $30 \times 10^{6}$ values of the energy, measured along a single MC run.

al., ${ }^{11}$ who reported a line of second order transitions. The first order nature of these transitions for large anisotropies is in agreement with recent results for a corresponding Ising model with dipolar interactions. ${ }^{8}$ Furthermore, as Fig. 1 shows, this line seems to go asymptotically, for large $\eta$, to a value of the transition temperature $T \approx 1.2$, which is in good agreement with the phase diagram of the Ising model. ${ }^{18}$ This quantitative agreement gives further credit to the first order nature of this transition line, at least for large $\eta$, where the Ising approximation is justified. More or less direct experimental evidence for the appearance of striped magnetic structures was reported already in a previous work by Allenspach and Bischof. ${ }^{3}$ More recently, the striped nature of the low temperature phase of high anisotropy, perpendicular $\mathrm{Fe} / \mathrm{Cu}(100)$ films, together with the transition to a phase with tetragonal symmetry, has been measured and confirmed by Vaterlaus et al. ${ }^{6} \mathrm{~A}$ theoretical model predicting the existence of a phase with $90^{\circ}$ symmetry induced by the underlying symmetry of the lattice was put forward by Abanov et al. ${ }^{14}$ The theory of Abanov et al. works in the Ising limit, where only the perpendicular component of the magnetization is relevant for the thermodynamic behavior. Their model admits two possible scenarios for the disordering of the stripes: one is similar to the present results with a first order transition from a stripe phase, with positional order decaying algebraically with distance to a paramagnetic phase with a residual $90^{\circ}$ symmetry, and a second possibility, depending on the values of elastic constants of the theory, in which an intermediate nematic phase (with orientational order and exponentially decaying positional order) appears between the stripes and paramagnetic phases. Furthermore, the perpendicular tetragonal phase can evolve continuously to a full paramagnetic phase or it can finish at a spin reorientation transition. Interestingly, our phase diagram shows these two behaviors (see Fig. 1): for an interval $6.7 \leqslant \eta \leqslant 7$, the Heisenberg system goes from stripes to tetragonal and then to a planar ferromagnet through a SRT. At still higher temperatures, the in-plane ferromagnet disorders via a continuous transition. This kind of behavior was already reported in experiments on $\mathrm{Fe} / \mathrm{Cu}(100)$ ultrathin films by Pappas et al., ${ }^{2}$ who found a gap in magnetization between the perpendicular and planar phases (see Fig. 1 of Ref. 2). Nevertheless, in (a)
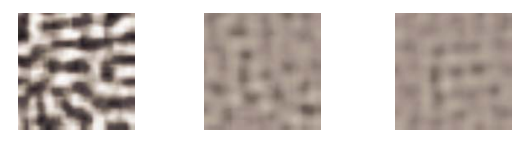

(b)

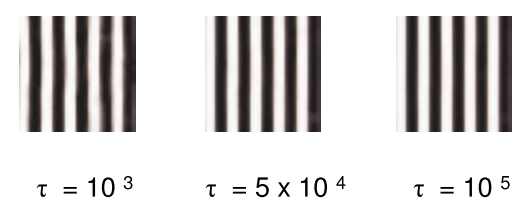

FIG. 3. (Color online) Time average of the local perpendicular magnetization $\overline{m_{\tau}}(\vec{r})$ at different average times $\tau$ (all times are measured in MCS) for $\delta=3, \eta=6.9$, and $L=40$. Before calculating $m_{\tau}(\vec{r})$, the system was thermalized for $t=10^{5}$ MCS at each temperature: (a) $T=1.1$ (tetragonal liquid phase) and (b) $T=0.9$ (striped phase).

those early experiments, the nature of the gap was not clear and the authors pointed out two possibilities: a real paramagnetic gap or the fact that the width of the stripes (not seen in the experiment) could diminish rapidly in the region of the SRT. One must note that the perpendicular phase in that series of experiments referred to samples with finite magnetization at low temperatures, not stripe order. Indeed, further experiments by Allenspach and Bischof ${ }^{3}$ confirmed the second hypothesis for the same range of thickness as that by Pappas et al. More recently, Won et al. ${ }^{5}$ analyzed domain formation and the nature of the SRT in ultrathin films of $\mathrm{Fe} / \mathrm{Ni} / \mathrm{Cu}(001)$ by using high resolution photoemision electron microscopy imaging techniques. They observed both kind of behaviors, according to the film thickness range, namely, a direct SRT from the striped state and a transition mediated by a paramagnetic gap. In this case, the resolution of the experiment rules out the possibility of domains with a stripe width below the magnetic spatial resolution in the gap region. However, as the authors pointed out, the possibility of a fast-moving striped domain phase cannot be excluded in that experiment. A direct inspection of the typical spin configurations (see snapshot in Fig. 1) indicates that in our simulations, the gap corresponds to an out-of-plane tetragonal phase appearing between the perpendicular stripe and planar ferromagnetic phases. Nevertheless, in experiments, only temporal averages can be observed. To emulate the acquisition image process of the experiments, we calculated a time average of the local magnetization (perpendicular component)

$$
\bar{m}_{\tau}(\vec{r}) \equiv \frac{1}{\tau} \sum_{t=1}^{\tau} S^{z}(\vec{r}, t)
$$

for different values of the "acquisition time" $\tau$, where all the times are measured in MCS. In Fig. 3, we show $\overline{m_{\tau}}(\vec{r})$ at three different values of $\tau$ in the striped and tetragonal liquid phases. The loss of contrast in the tetragonal liquid phase for relatively short times $\tau$ shows that the characteristic time scales for the fluctuations in this phase are much shorter than in the striped phase. 


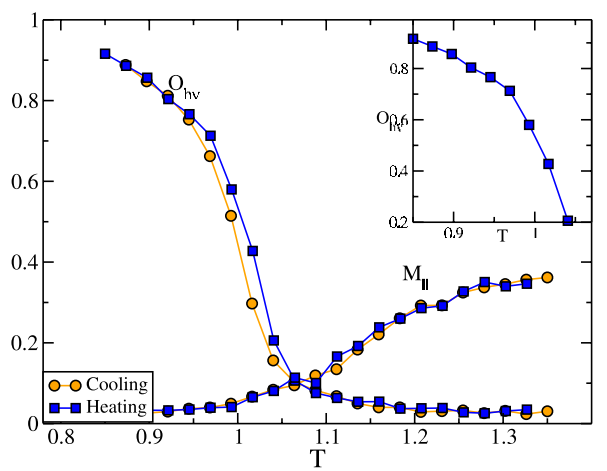

FIG. 4. (Color online) Order parameter $O_{h v}$ and in-plane magnetization $M_{\|}$as a function of temperature for $\delta=3, \eta=6.9$. The system was first thermalized at $T=1.4$ and cooled with a rate $r$ $=10^{-7}$, and then heated again with the same rate. The error bars are of the same order of the symbol size. The inset shows a zoom of the heating curve.

In Fig. 4, we show the orientational order parameter $O_{h v}$ and in-plane magnetization $M^{\|}$for $\eta=6.9$ as a function of temperature. Figure 4 was obtained by performing cooling and heating cycles at a very small cooling rate $r=10^{-7}$. Note that the stripe-tetragonal transition shows weak hysteresis. This indicates that the transition may be weakly first order or even continuous in this region. To give a definite answer, one must simulate larger samples and obtain much better statistics. Nevertheless, it is clear from these curves that the sharp first order transition present for higher values of the anisotropy is much weaker in this region. Notice also the presence of a small shoulder in the orientational order parameter (see inset of Fig. 4). This effect is more marked in many individual realizations of the stochastic noise, where an almost saturated value of $O_{h v}$ smaller than 1 can be observed in a narrow range of temperatures below the transition temperature. The same effect appears for larger values of $\eta$. This opens the possibility for the second scenario predicted by Abanov et al. ${ }^{14}$ of an intermediate perpendicular nematic phase with long range orientational order but without positional order. Evidence for this phase comes also from simulations of the Ising dipolar model ${ }^{9}$ and a recent theoretical model for the nematic transition in two-dimensional systems with competing interactions. ${ }^{12,13}$

\section{B. Spin reorientation transition}

In the region between $\eta=5.8$ and $\eta=7.0$, we directly observe a sharp SRT from a perpendicular stripe phase to a planar ferromagnetic one. The behavior of the orientational order parameter and the in-plane magnetization with temperature is shown in Fig. 5 for $\eta=6.5$. In this region, there is no gap between the perpendicular and in-plane phases.

The SRT can be accessed both by varying the temperature in a film of fixed thickness and by varying the thickness $d$ of the film at fixed temperature. In fact, as the thickness grows, the in-plane anisotropy induced by the dipolar interactions is reinforced, while the perpendicular anisotropy stays nearly constant due to its essentially surface character. Conse-

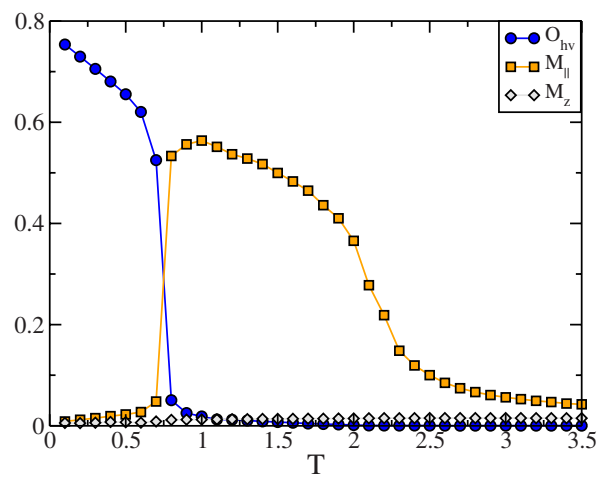

FIG. 5. (Color online) Order parameter $O_{h v}$ and in-plane and out-of-plane magnetizations, $M_{\|}$and $M_{z}$, as a function of temperature for $\delta=3$ and $\eta=6.5$. The system was initialized at infinite temperature, thermalized at $T=6$, and then cooled (equilibrated at each temperature).

quently, at some thickness, a SRT can be observed. Then, it is reasonable to consider a phenomenological model where the thickness acts equivalently to the inverse anisotropy: $d$ $\propto 1 / \eta$. In fact, Won et al. ${ }^{5}$ reported detailed measurements of magnetic changes as the thickness or temperature of samples of $\mathrm{Fe} / \mathrm{Ni} / \mathrm{Cu}(001)$ changed. They rationalized the observed behavior through a phenomenological model and summarized their findings in a phase diagram showing "temperature versus Fe thickness" (Fig. 5 of Ref. 5). By assuming an approximate equivalence between thickness and inverse anisotropy, as explained above, we plotted our simulation data in a " $T$ versus $1 / \eta$ " diagram, as shown in Fig. 6 . This figure shows a striking similarity with the right half of Fig. 5 of Won et al., reinforcing the equivalent character of film width and anisotropy in these systems.

The order of appearance of the perpendicular and planar phases is the main difference between our results and a previous phase diagram for the same model obtained by MacIsaac et al. ${ }^{11}$ Those authors obtained a SRT line in the reverse order, from perpendicular at high temperatures to planar at low temperatures. As shown above, the correctness of our results is supported by experimental evidence on different ultrathin films as well as by a theoretical analysis on

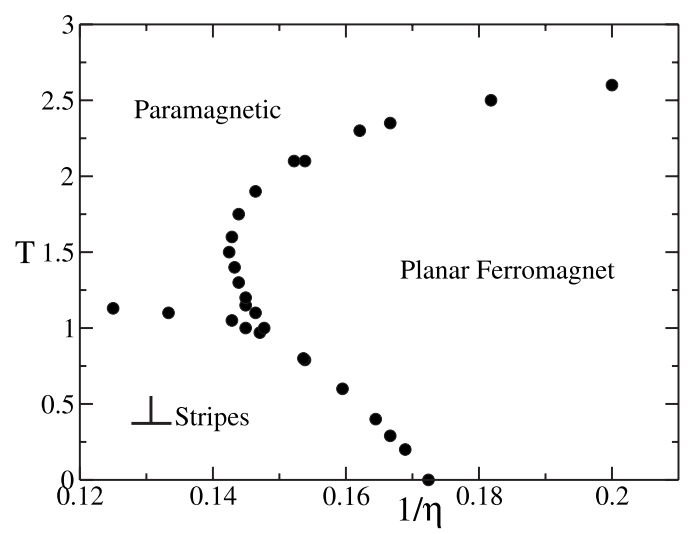

FIG. 6. Phase diagram showing temperature versus inverse anisotropy. 


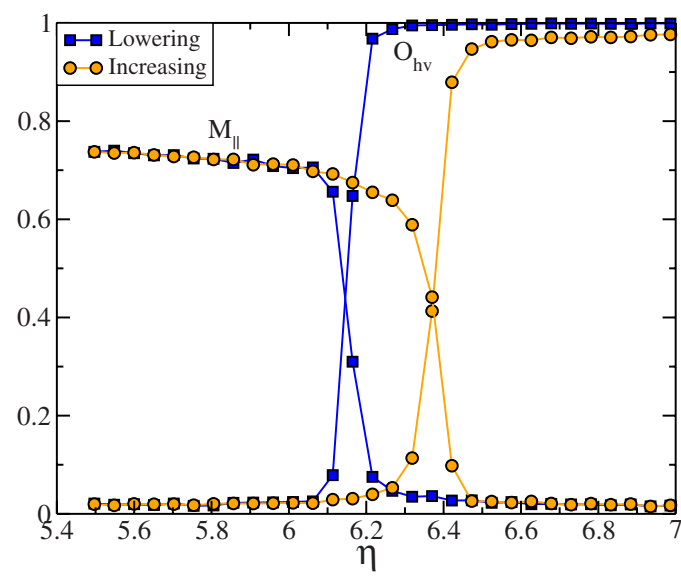

FIG. 7. (Color online) Order parameter $O_{h v}$ and in-plane magnetization $M_{\|}$as a function of $\eta$ for $\delta=3$ and $T=0.6$. The system was initialized in stripes of width 4 , thermalized at $\eta=7.5$, and then $\eta$ was lowered and then increased with a rate of $r=10^{-5}$.

the effect of thermal fluctuations on the SRT at fixed film thickness. Thermal fluctuations renormalize the dipolar and anisotropy coupling parameters in such a way that the anisotropy $K(T)$ diminishes faster than the dipolar coupling constant $^{19,20} g(T)$ (in our notation, $\eta=K / g$ ). Those works predict a linear dependence of the transition temperature $T_{S R T}(\eta)$ with anisotropy with positive slope, which is roughly in agreement with our SRT line from Monte Carlo simulations. In Fig. 7, we show cycles of $O_{h v}$ and $M^{\|}$varying $\eta$ at a fixed temperature of $T=0.6$ in the SRT region. The cycles show a strong hysteretic behavior. This is further confirmed by means of energy histograms shown in Fig. 8, which show again the change in stability between the perpendicular and planar phases, a signature of the first order nature of the SRT, as predicted theoretically by several authors. ${ }^{19,21}$

\section{Planar ferromagnetic-paramagnetic transition}

This transition line shows a maximum around $\eta=7, T$ $=1.5$ in the $(\eta, T)$ plane. One can expect that the behavior of

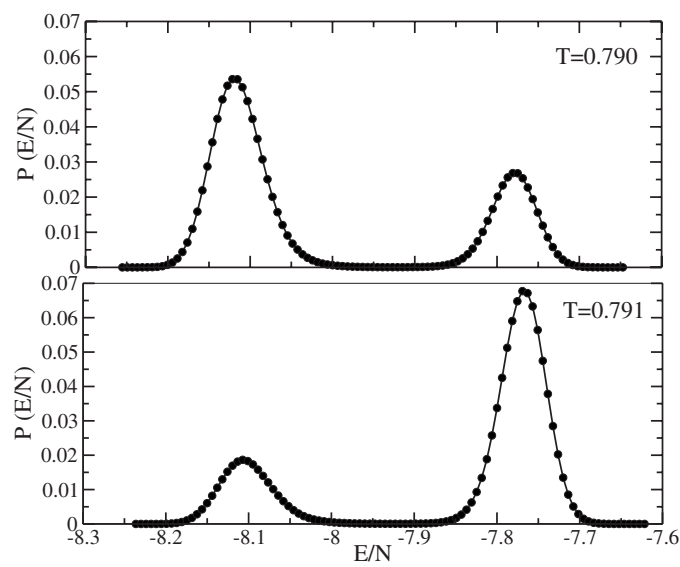

FIG. 8. Energy per spin histograms for $\delta=3, \eta=6.5$, and $T$ $=0.790$ (up) and $T=0.791$ (down). The histograms were calculated for $30 \times 10^{6}$ energies measured along a single MC run.

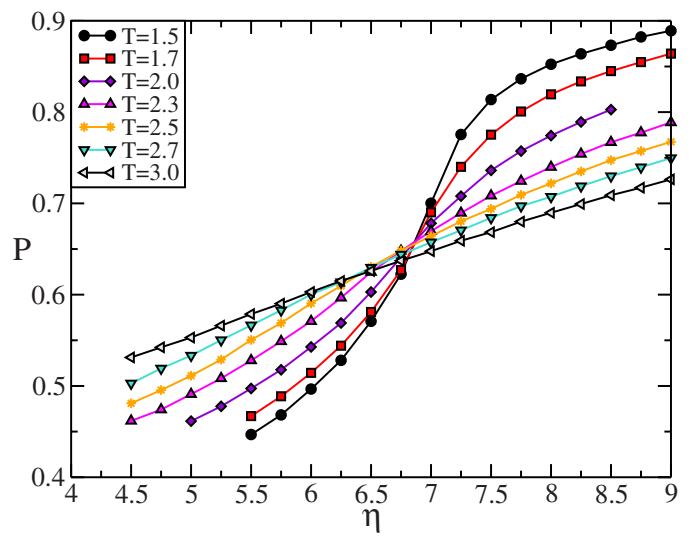

FIG. 9. (Color online) Mean absolute magnetization $P$ for different temperatures as function of $\eta$ across the planar ferroparamagnetic line $(\delta=3$ and $L=24)$.

the system across the transition line will be different in the regions to the right and to the left of the maximum point. In the far right, the system goes continuously from an in-plane ferromagnet to a paramagnetic phase. We have not been able to characterize completely the nature of this transition, although our results appear to be consistent with a second order one.

In Fig. 9, we show that already at small values of $\eta$, deep in the planar phase, the spins have a finite perpendicular component, which grows continuously with $\eta$ as the system goes through the phase transition. At some point around $\eta$ $=\eta_{c} \approx 6.8$, the curves cross in an inflection point upon which the perpendicular component tends to saturate. This value of $\eta$ drifts toward slightly smaller values as the temperature increases. We do not have a clear interpretation for the crossing points. It would be very interesting to analyze the domain walls in the paramagnetic phase and how they influence the evolution of the perpendicular magnetization as the system goes through the phase transition with finite in-plane magnetization. One may naively expect the perpendicular component of the local magnetizations $m_{i}^{z}$ to vanish in this region, but this is not the case as Fig. 9 shows. Instead, at low temperatures and $\eta<\eta_{c}$, when the planar ferromagnetic phase is favored, the perpendicular component of the local magnetization does not vanish (implying $P$ to be nonzero), because the anisotropic Heisenberg model enables out-ofplane fluctuations at finite temperature. As temperature is increased, out-of-plane fluctuations become stronger and stronger, causing $P$ to increase, until they drive the system into the paramagnetic phase. On the other hand, at low temperatures and $\eta>\eta_{c}$, the perpendicular stripes are well defined (domain walls are sharp) so that $P$ is slightly smaller than 1. As temperature is increased, the system evolves into the tetragonal phase and then into the paramagnetic phase: the domain walls gradually soften so that $P$ decreases.

In Fig. 10, specific heat curves are shown for different anisotropies in the same region to the right of the maximum along the transition line. Note that the peak in the specific heat decreases as the transition approaches the maximum point from the right, suggesting a weakening of the second order character of the transition in this direction. In Fig. 11, 


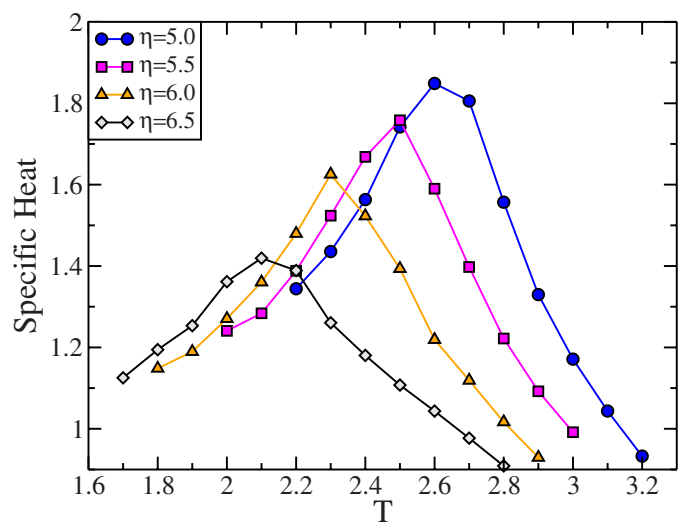

FIG. 10. (Color online) Specific heat for $\delta=3$ and different values of $\eta$.

we show a hysteresis cycle in $\eta$ of the parallel component of the magnetization for a temperature to the left of the maximum point of the curve. A very weak hysteresis effect is observed. This behavior is compatible with a continuous transition or even a weakly first order one. Note that above the transition line in this region, the system enters the tetragonal phase as discussed above. This phase has a different symmetry as compared to the paramagnetic high temperature phase. The change from the continuous rotational symmetry of the planar ferromagnetic phase to the discrete rotational symmetry of the tetragonal liquid would be compatible with a discontinuous phase transition in that part of the phase diagram. Also, notice that actually along this line there is also a SRT, because the tetragonal liquid phase is perpendicularly oriented. Since we already showed the first order nature of the SRT at planar ferromagnetic-striped transition line (where a similar change of symmetry happens), one would expect the planar ferromagnetic-tetragonal liquid line to present the same character. Indeed, a mean field analysis of a multilayered version of the model ${ }^{22}$ predicts first order SRT in the monolayer limit. The previous analysis seems to indicate that a different nature of the phase transition to the left and right of the maximum is possible, although more detailed studies are necessary in order to elucidate this point.

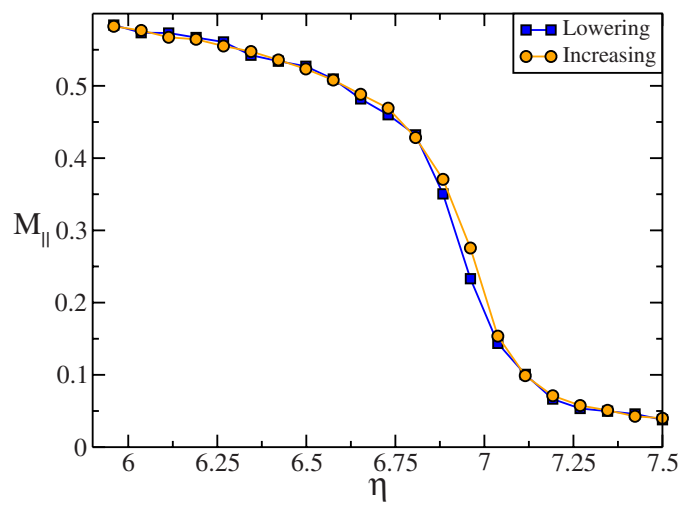

FIG. 11. (Color online) $M_{\|}$as a function of $\eta$ for a fixed temperature $T=1.3$.

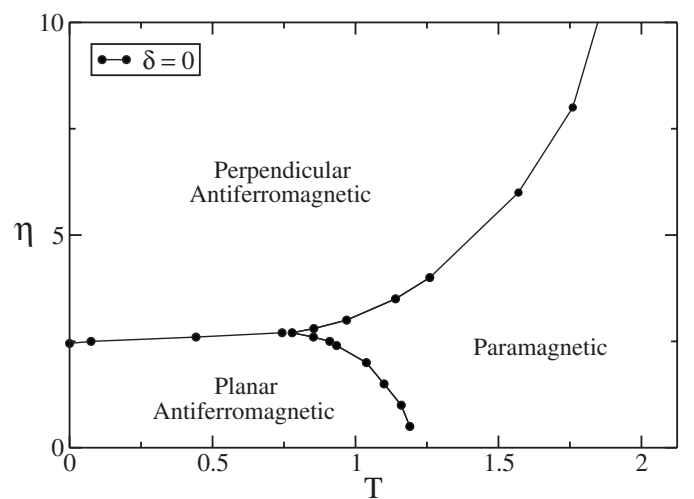

FIG. 12. Phase diagram for $\delta=0$ (pure dipolar plus anisotropy).

\section{PURE DIPOLAR PLUS ANISOTROPY FILM}

In this section, we briefly discuss results for the limit where exchange interactions are absent, $\delta=0$. Experimentally, this limit may be relevant to the behavior of arrays of magnetic monodomain particles for application in data storage devices. Usually these arrays can be considered as composed of noninteracting dots, but as the density of dots grows, dipolar effects may begin to be relevant to the magnetic behavior. Although relaxation effects of arrays of this type have been studied by several authors, much less is known on the thermodynamic properties of the system. In particular, MacIsaac et al. ${ }^{16}$ obtained a phase diagram by Monte Carlo simulations. Without exchange interactions, the relevant ordered phases in this case are all antiferromagnetic: one out-of-plane, with sublattice magnetization, and the other one in-plane. ${ }^{16,17}$ A SRT is also found in this limit, from a planar antiferromagnetic phase at small anisotropies to a perpendicular antiferromagnetic phase at large anisotropies. Similar to what happened in the $\delta \neq 0$ case, MacIsaac et al. also found a reverse order of appearance of the phases through the SRT with temperature (see Fig. 1 of Ref. 16). We obtained, instead, a different behavior, again similar to the trend of the $\delta \neq 0$ case, from perpendicular at low temperatures to planar at high temperatures, as shown in Fig. 12.

By comparing with Fig. 1, we note that the slope of the SRT line is very small. Nevertheless, there is a finite window where the transition from out-of-plane to in-plane sublattice magnetization is sharp, as can be seen in Fig. 13. Another important difference between the phase diagrams of Figs. 1 and 12 is the absence of the gap for any fixed anisotropy in the latter case. This may be related to the different symmetry of the phases in the pure dipolar case. Now there is no tetragonal phase and, for large anisotropies, the system goes directly from a perpendicular antiferromagnetic phase to a perpendicularly disordered phase with full rotational symmetry. In this sense, the paramagnetic phase shows the same symmetry along the whole planar-paramagnetic line at variance with the corresponding line in the ferromagnetic case.

\section{CONCLUSIONS}

In this work, we have analyzed the finite temperature phase diagram of a model for ultrathin ferromagnetic films 


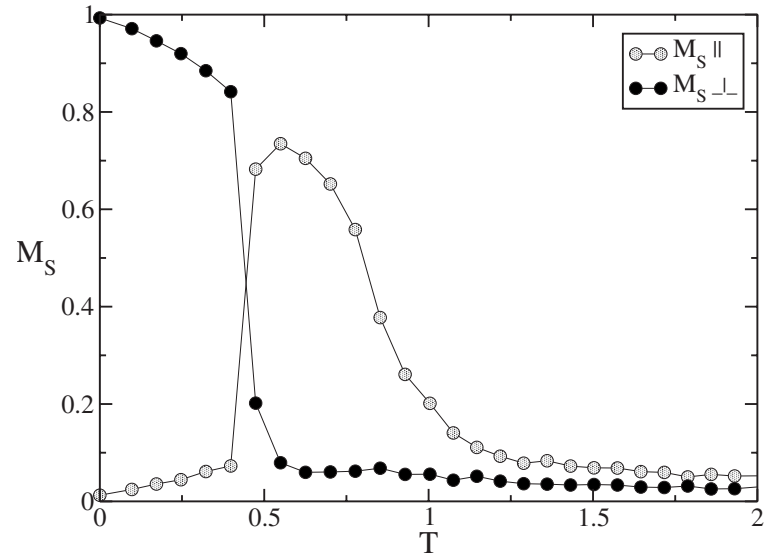

FIG. 13. Order parameters as function of temperature for $\delta=0$ and $\eta=2.6$. Here, the system size is $L=32$.

with exchange, dipolar, and perpendicular anisotropy interactions for two different values of the exchange constant (relative to the dipolar one). Particular emphasis was put in the $\delta=3$ case, where the system presents a striped phase of width $h=4$ at low temperatures and a SRT to a planar ferromagnetic phase as the temperature increases. Although we were not able to simulate systems with larger (and more realistic) values of the exchange constant, the overall qualitative good agreement with many experimental results indicates that the same global behavior should be expected. In particular, the comparison between our phase diagram and the temperature vs film thickness of Won et al. ${ }^{5}$ for $\mathrm{Fe} / \mathrm{Ni} / \mathrm{Cu}$ films suggests that the film thickness acts as an effective inverse anisotropy. We also reproduced the gap between the striped and planar phases found by Won et al. Moreover, our results seem to indicate that the physical origin of the gap relies on the presence of a fast-moving perpendicularly oriented labyrinthine (tetragonal liquid) phase. Evidence of a similar phenomenon (a fast-moving striped phase close to the order-disorder transition) in $\mathrm{Fe}$ on $\mathrm{Cu}$ films has been reported by Portmann et al. ${ }^{23}$
Concerning the thermodynamical nature of the different transitions involved in the phase diagram, we obtained a clear numerical evidence of a first order stripe-planar SRT at low temperatures. We also found evidence pointing toward a first order nature of the stripe-tetragonal liquid transition, consistent with previous results in the Ising (i.e., high anisotropy) limit. 8,9

The planar ferromagnet-disordered transition line presents a maximum in the $(\eta, T)$ space. In the left part of this line, the disordered state is a tetragonal liquid state, while in the right part, we have a transition to an isotropic paramagnetic state; above the maximum, the system passes continuously (i.e., without any thermodynamical phase transition) from the tetragonal liquid to the paramagnet. This fact, together with several other physical arguments, suggests the possibility of a change in the order of the transition around the maximum of the line, being weakly first order in the left part of the line and second order in the right part. If confirmed, this would imply the existence of a tricritical point around the maximum and a triple point where the three phases (stripeplanar-tetragonal) coexist. However, strong finite size effects did not allow us to give a definite answer concerning this point and further studies are needed.

In the case of $\delta=0$, we showed the existence of a SRT from a perpendicular antiferromagnetic phase at low temperature to an in-plane antiferromagnetic phase at higher temperatures, at variance with previous reported results. The present results suggest that a SRT from a low temperature out-of-plane to an in-plane phase at higher temperatures for low values of $\eta$ is present for any value of $\delta$.

\section{ACKNOWLEDGMENTS}

This work was partially supported by grants from CONICET (Argentina), SeCyT, Universidad Nacional de Córdoba (Argentina), CNPq (Brazil), FONCyT Grant No. PICT-200533305 (Argentina), and ICTP Grant No. NET-61 (Italy).

\footnotetext{
*carubell@famaf.unc.edu.ar

†billoni@famaf.unc.edu.ar

†spighin@famaf.unc.edu.ar

§cannas@famaf.unc.edu.ar

"stariolo@if.ufrgs.br

Itamarit@famaf.unc.edu.ar

${ }^{1}$ O. Portmann, Micromagnetism in the Ultrathin Limit (Logos Verlag, Berlin, 2006).

${ }^{2}$ D. P. Pappas, K. P. Kamper, and H. Hopster, Phys. Rev. Lett. 64, 3179 (1990).

${ }^{3}$ R. Allenspach and A. Bischof, Phys. Rev. Lett. 69, 3385 (1992).

${ }^{4}$ Y. Yafet and E. M. Gyorgy, Phys. Rev. B 38, 9145 (1988).

${ }^{5}$ C. Won, Y. Z. Wu, J. Choi, W. Kim, A. Scholl, A. Doran, T. Owens, J. Wu, X. F. Jin, H. W. Zhao and Z. Q. Qiu, Phys. Rev. B 71, 224429 (2005).

${ }^{6}$ A. Vaterlaus, C. Stamm, U. Maier, M. G. Pini, P. Politi, and D. Pescia, Phys. Rev. Lett. 84, 2247 (2000).
}

${ }^{7}$ A. B. MacIsaac, J. P. Whitehead, M. C. Robinson, and K. De'Bell, Phys. Rev. B 51, 16033 (1995).

${ }^{8}$ S. A. Cannas, D. A. Stariolo, and F. A. Tamarit, Phys. Rev. B 69, 092409 (2004).

${ }^{9}$ S. A. Cannas, M. F. Michelon, D. A. Stariolo, and F. A. Tamarit, Phys. Rev. B 73, 184425 (2006).

${ }^{10}$ E. Rastelli, S. Regina, and A. Tassi, Phys. Rev. B 73, 144418 (2006).

${ }^{11}$ A. B. MacIsaac, K. De'Bell, and J. P. Whitehead, Phys. Rev. Lett. 80, 616 (1998).

${ }^{12}$ L. Nicolao and D. A. Stariolo, Phys. Rev. B 76, 054453 (2007).

${ }^{13}$ D. G. Barci and D. A. Stariolo, Phys. Rev. Lett. 98, 200604 (2007).

${ }^{14}$ A. Abanov, V. Kalatsky, V. L. Pokrovsky, and W. M. Saslow, Phys. Rev. B 51, 1023 (1995).

${ }^{15}$ I. Booth, A. B. MacIsaac, J. P. Whitehead, and K. De'Bell, Phys. Rev. Lett. 75, 950 (1995). 
${ }^{16}$ A. B. MacIsaac, J. P. Whitehead, K. De'Bell, and P. H. Poole, Phys. Rev. Lett. 77, 739 (1996).

${ }^{17}$ K. De'Bell, A. B. MacIsaac, I. N. Booth, and J. P. Whitehead, Phys. Rev. B 55, 15108 (1997).

${ }^{18}$ S. A. Pighin and S. A. Cannas, Phys. Rev. B 75, 224433 (2007).

${ }^{19}$ D. Pescia and V. L. Pokrovsky, Phys. Rev. Lett. 65, 2599 (1990).
${ }^{20}$ P. Politi, A. Rettori, and M. G. Pini, Phys. Rev. Lett. 70, 1183 (1993).

${ }^{21}$ P. Politi, Comments Condens. Matter Phys. 18, 191 (1998).

${ }^{22}$ A. Moschel and K. D. Usadel, Phys. Rev. B 51, 16111 (1995).

${ }^{23}$ O. Portmann, A. Vaterlaus, and D. Pescia, Phys. Rev. Lett. 96, 047212 (2006). 